\title{
Effect of Resonant Continuum on Pairing Correlations in the Relativistic Approach
}

\author{
Li-Gang Cao \\ Institute of High Energy Physics, Chinese Academy of Sciences, Beijing 100039 \\ Zhong-Yu Ma* \\ CCAST, Beijing 100080, P.R. of China and \\ China Institute of Atomic Energy, Beijing 102413, P.R. of China
}

(Dated: September 13, 2018)

\begin{abstract}
A proper treatment of the resonant continuum is to take account of not only the energy of the resonant state, but also its width. The effect of resonant states on pairing correlations is presented in the framework of the relativistic mean field theory plus Bardeen-Cooper-Schrieffer (BCS) approximation with a constant pairing strength. The study is performed in an effective Lagrangian with the parameter set NL3 for neutron rich even-even Ni isotopes. Results show that the contribution of the proper treatment of the resonant continuum to pairing correlations for those nuclei close to neutron drip line is important. The pairing gaps, Fermi energies, pairing correlation energies, and binding energies are considerably affected with a proper consideration of the width of resonant states. The problem of unphysical particle gas, which may appear in the calculation of the traditional mean field plus BCS method for nuclei in the vicinity of drip line could be well overcome when the pairing correlation is performed by using the resonant states instead of the discretized states in the continuum.

PACS numbers: 21.60.-n Nuclear structure models and methods 24.10.Jv Relativistic models
\end{abstract}

\footnotetext{
*also Center of Theoretical Nuclear Physics, National Laboratory of Heavy Ion Accelerator of Lanzhou, Lanzhou 730000, P. R. of China and Institute of Theoretical Physics, Beijing 100080, P.R. of China
} 


\section{INTRODUCTION}

The study of properties of exotic nuclei, in particular the structure of nuclei far from the $\beta$-stability line, has recently attracted wide interest both experimentally and theoretically. In those nuclei, the presence of nucleons, which have separation energies appreciably smaller than those in $\beta$-stable nuclei and have a far extending nucleon distribution, leads to various interesting physical phenomena. Due to the closeness of the Fermi surface to the particle continuum in exotic nuclei the coupling between bound states and the continuum become important. The description of exotic nuclei has to explicitly include the coupling between bound states and the continuum. There exist several extensive studies of exotic nuclei in relativistic and non-relativistic microscopic approaches 1, 2, 3, 4]. A key ingredient of those models is how to properly treat the pairing correlations which have an important influence on physical properties in exotic nuclei. In general, pairing correlations in open shell nuclei can be treated by the Bardeen-Cooper-Schrieffer (BCS) theory or through the Bogoliubov transformation [5]. The main feature of the Bogoliubov transformation, compared with the simple BCS theory, is that the Hartree-Fock equation or Dirac equation and the gap equations are solved simultaneously with self-consistent fields. Both of them could give a good description of the pairing correlation if the nucleus is not too far away from the $\beta$-stable line 6]. The simple BCS method may not be reliable near the drip line because the continuous states were not correctly treated 6 , 7]. The contribution of the coupling to the continuum would be prominent when the nucleus close to the drip line, therefore a proper treatment of the continuum becomes more important. Single particle resonant states in the continuum, which differ from bound states, are metastable states with life times due to a sufficiently high centrifugal barrier (for neutrons and protons) and Coulomb barrier (for protons). The effect of the widths of resonant states on pairing correlations was not estimated by the usual non-relativistic Hartree-Fock-Bogoliubov (HFB) 4, 5] or the relativistic Hartree-Bogoliubov (RHB) 8]. The effect of a proper treatment of the resonant continuum on the pairing in the non-relativistic approach has been investigated 9 , 10, 11]. The investigation in the relativistic approach is reported very recently 12$]$.

In this paper, we aim at the investigation on the effect of the resonant continuum on pairing correlations of neutron rich nuclei in the relativistic mean field theory plus BCS $(\mathrm{RMF}+\mathrm{BCS})$ approximation. Particularly we shall focus our attention on the effect of 
widths of single particle resonant states in the description of neutron rich nuclei. It has been pointed out that pairing correlations could be well described by the simple BCS theory if single particle states in the continuum are properly treated $[9,10,11]$. It is known that narrow resonant states in the continuum are well localized inside the nucleus due to a sufficiently high centrifugal barrier and Coulomb barrier (for proton only). In our previous work [13], we have shown that the nuclear dynamical processes can also be well described by the simple method where the continuum was replaced by only a few single particle resonant states. Therefore, we may expect that using a few single particle resonant states instead of the discretized continuum, the pairing space would be large enough in order to give a good description on the ground state properties of neutron rich nuclei in the RMF, even within the BCS approximation.

Usually pairing correlations in the BCS approximation are treated in a phenomenological way based on empirical pairing gaps deduced from odd-even mass differences. This procedure works well in the valley of $\beta$-stability, where experimental masses are known. The predictive power for pairing gaps for nuclei far from the $\beta$-stability line is thus limited due to the unknown masses of such nuclei. Therefore, a constant pairing strength is usually employed in the BCS calculation 14]. We shall investigate the pairing effect in the RMF with the parameter set NL3 [15], which give a good description of not only the ground state properties [16] but also the collective giant resonance 17, 18, 19, 20]. To investigate the width effect of single particle resonant states on pairing correlations, we compare the results in three types of calculations: (a) the RMF+BCS approach where quasi-particle wave functions are obtained by a box boundary condition; (b) the extended RMF + BCS approximation where wave functions of resonant states are calculated by imposing a proper scattering boundary condition for the continuous spectrum but the width of resonant states is not considered, which is denoted as RMF+BCSR; (c) the full RMF+BCS (RMF+BCSRW) calculation where widths of single particle resonant states are taken into account explicitly.

The paper is arranged as the follows. In Sec.II and Sec.III the relativistic mean field theory and the BCS approximation with explicitly considering the widths of the resonant continuum are presented. The effect of the resonant continuum on pairing correlations is formulated by introducing a continuum level density into the gap equations. The results calculated by different models mentioned above for neutron rich even-even $\mathrm{Ni}$ isotopes are given and discussed in Sec.IV. Finally we give a brief summary in Sec.V. 


\section{RELATIVISTIC MEAN FIELD THEORY}

The RMF theory [1, 21, 22] is based on the effective Lagrangian density which includes the nucleon field $(\psi)$, the isoscalar scalar meson field $(\sigma)$, the isoscalar vector meson field $(\omega)$, the isovector vector meson field $(\boldsymbol{\rho})$, and electromagnetic field $(A)$. The Lagrangian density is expressed as the following:

$$
\begin{aligned}
\mathcal{L}= & \bar{\psi}\left(i \gamma^{\mu} \partial_{\mu}-M\right) \psi+\frac{1}{2} \partial_{\mu} \sigma \partial^{\mu} \sigma-U(\sigma)-\frac{1}{4} \Omega_{\mu \nu} \Omega^{\mu \nu} \\
& +\frac{1}{2} m_{\omega}^{2} \omega_{\mu} \omega^{\mu}-\frac{1}{4} \boldsymbol{R}_{\mu \nu} \boldsymbol{R}^{\mu \nu}+\frac{1}{2} m_{\rho}^{2} \boldsymbol{\rho}_{\mu} \boldsymbol{\rho}^{\mu}-\frac{1}{4} F_{\mu \nu} F^{\mu \nu} \\
& -g_{\sigma} \bar{\psi} \sigma \psi-g_{\omega} \bar{\psi} \gamma^{\mu} \omega_{\mu} \psi-g_{\rho} \bar{\psi} \gamma^{\mu} \boldsymbol{\rho}_{\mu} \cdot \boldsymbol{\tau} \psi \\
& -e \bar{\psi} \gamma^{\mu} A_{\mu}\left(\frac{1-\tau_{3}}{2}\right) \psi
\end{aligned}
$$

where $M, m_{\sigma}, m_{\omega}$, and $m_{\rho}$ denote the nucleon, $\sigma, \omega$, and $\rho$ mesons masses, respectively, while $g_{\sigma}, g_{\omega}$ and $g_{\rho}$ are the corresponding coupling constants for the mesons, respectively. The vectors in isospin space are denoted by bold-faced symbols. $\Omega^{\mu \nu}, \boldsymbol{R}^{\mu \nu}$ and $F^{\mu \nu}$ are the field tensors of the vector fields $\omega, \rho$ and of the photon, respectively, they are defined as:

$$
\begin{aligned}
& \Omega^{\mu \nu}=\partial^{\mu} \omega^{\nu}-\partial^{\nu} \omega^{\mu}, \\
& \boldsymbol{R}^{\mu \nu}=\partial^{\mu} \boldsymbol{\rho}^{\nu}-\partial^{\nu} \boldsymbol{\rho}^{\mu}, \\
& F^{\mu \nu}=\partial^{\mu} A^{\nu}-\partial^{\nu} A^{\mu},
\end{aligned}
$$

In the Eq.(1), a non-linear scalar self-interaction term [23] $U(\sigma)$ of the $\sigma$ meson has been taken into account:

$$
U(\sigma)=\frac{1}{2} m_{\sigma}^{2} \sigma^{2}+\frac{1}{3} g_{2} \sigma^{3}+\frac{1}{4} g_{3} \sigma^{4}
$$

The Dirac equation of a single-particle state in the RMF approximation can be expressed as following:

$$
[\boldsymbol{\alpha} \cdot \boldsymbol{p}+V(r)+\beta(M-S(r))] \psi_{\alpha}=E_{\alpha} \psi_{\alpha}
$$

where $E_{\alpha}$ and $\psi_{\alpha}$ are the single-particle energy and wave function, respectively. $S(r)$ and $V(r)$ are an attractive scalar and repulsive vector potential, respectively. In the RMF approximation, scalar and vector potentials are produced by the classical meson fields: isoscalar 
$\sigma, \omega$ and isovector $\rho$ mesons as well as the photon, which are obtained in a self-consistent calculation for the nuclear ground state $1,21,22]$. The nucleon spinor $\psi_{\alpha}$ in the Dirac equation is expressed as:

$$
\psi_{\alpha}(\boldsymbol{r})=\frac{1}{r}\left(\begin{array}{c}
i G_{a}(r) \\
F_{a}(r) \boldsymbol{\sigma} \cdot \hat{\boldsymbol{r}}
\end{array}\right) \Phi_{\kappa, m}(\hat{r}) \chi_{\frac{1}{2}}
$$

where $G_{a}$ and $F_{a}$ are upper and lower components of nucleon wave function, $\chi_{\frac{1}{2}}$ is the isospinor and $\Phi_{\kappa, m}$ is a spherical harmonics function. $\alpha$ is a set of quantum numbers $\alpha=$ $\left(n, l, j, m, \tau_{3}\right) \equiv\left(a, m, \tau_{3}\right) . \kappa$ is the Dirac quantum number given by:

$$
\kappa=\left\{\begin{array}{l}
-\left(j+\frac{1}{2}\right), \text { for } j=l+\frac{1}{2} \\
+\left(j+\frac{1}{2}\right), \text { for } j=l-\frac{1}{2}
\end{array} .\right.
$$

For spherical nuclei, the Dirac equation can be reduced to coupled equations of the radial part for $G_{a}(r)$ and $F_{a}(r)$ :

$$
\frac{d}{d r}\left(\begin{array}{c}
G_{a}(r) \\
F_{a}(r)
\end{array}\right)=\left(\begin{array}{cc}
-\frac{\kappa}{r} & \left.M+E_{a}-S(r)-V(r)\right) \\
M-E_{a}-S(r)+V(r) & \frac{\kappa}{r}
\end{array}\right)\left(\begin{array}{c}
G_{a}(r) \\
F_{a}(r)
\end{array}\right),
$$

where $S(r)$ is the scalar potential:

$$
S(r)=g_{\sigma} \sigma(r)
$$

and $V(r)$ denotes the vector potential:

$$
V(r)=g_{\omega} \omega_{0}(r)+g_{\rho} \tau_{3} \rho_{0}(r)+e \frac{1-\tau_{3}}{2} A_{0}(r),
$$

The meson and electromagnetic fields obey the radial Laplace equations:

$$
\begin{gathered}
\frac{d^{2}}{d r^{2}} \sigma(r)+\frac{2}{r} \frac{d}{d r} \sigma(r)-m_{\sigma}^{2} \sigma(r)=-g_{\sigma} \rho_{s}(r)-g_{2} \sigma^{2}(r)-g_{3} \sigma^{3}(r), \\
\frac{d^{2}}{d r^{2}} \omega_{0}(r)+\frac{2}{r} \frac{d}{d r} \omega_{0}(r)-m_{\omega}^{2} \omega_{0}(r)=-g_{\omega} \rho_{v}(r), \\
\frac{d^{2}}{d r^{2}} \rho_{0}(r)+\frac{2}{r} \frac{d}{d r} \rho_{0}(r)-m_{\rho}^{2} \rho_{0}(r)=-g_{\rho} \rho_{3}(r), \\
\frac{d^{2}}{d r^{2}} A_{0}(r)+\frac{2}{r} \frac{d}{d r} A_{0}(r)=-e \rho_{c}(r)
\end{gathered}
$$


with

$$
\begin{gathered}
\rho_{s}(r)=\sum_{a}^{o c c} \frac{2 j_{a}+1}{4 \pi}\left(\left|G_{a}(r)\right|^{2}-\left|F_{a}(r)\right|^{2}\right) \\
\rho_{v}(r)=\sum_{a}^{o c c} \frac{2 j_{a}+1}{4 \pi}\left(\left|G_{a}(r)\right|^{2}+\left|F_{a}(r)\right|^{2}\right), \\
\rho_{3}(r)=\sum_{a}^{o c c} \frac{2 j_{a}+1}{4 \pi} \tau_{3}\left(\left|G_{a}(r)\right|^{2}+\left|F_{a}(r)\right|^{2}\right), \\
\rho_{c}(r)=\sum_{a}^{o c c} \frac{2 j_{a}+1}{4 \pi}\left(\frac{1-\tau_{3}}{2}\right)\left(\left|G_{a}(r)\right|^{2}+\left|F_{a}(r)\right|^{2}\right),
\end{gathered}
$$

The sums a run over all occupied states. The Dirac equation Eq.(9) and the corresponding meson field functions Eq.(12 - 14) as well as the photon field function Eq.(15) with expressions for source terms $(16$ - 19) can be solved self-consistently. More details on the relativistic mean-field theory can be found in Refs. 1, 21, 22].

\section{BCS APPROXIMATION WITH RESONANT CONTINUUM}

It is well known that the pairing correlation plays an important role in describing the ground state properties of open shell nuclei. The non-relativistic HFB 4, 5] or RHB 8] theory can provide a unified description on the mean field and pairing correlations, but they require much more numerical effort than the simple BCS theory. However, the width effect of resonant states in the gap equation is missing in most previous works of the BCS theory or the Bogoliubov transformation. Recently, properly treating the contribution of resonant continuum to pairing correlations has been investigated in the non-relativistic $\mathrm{HF}+\mathrm{BCS}$ as well as HFB in Refs. [9, 10, 11, 24]. It has been pointed out [24] that the resonant continuum $\mathrm{HF}+\mathrm{BCS}$ approximation with a proper treatment of resonant continuum can reproduce the pairing correlation energies predicted by the continuum HFB approach up to the drip line. In this paper, we investigate the width effect of single particle resonant states on the pairing correlations in the relativistic approach. The pairing correlations of open shell nuclei are treated based on the RMF within the BCS approximation. A constant pairing strength is adopted in the BCS theory. Although the extended RMF+BCS theory formulated with correct boundary conditions for the continuous spectrum is available in the literature 12], in the following we briefly summarize the essential points together with our model. From 
Ref. [5] , assuming constant pairing matrix elements in the vicinity of Fermi level, one can obtain the so-called gap equations:

$$
\begin{gathered}
\sum_{a}\left(j_{a}+\frac{1}{2}\right) \frac{1}{\sqrt{\left(\varepsilon_{a}-\lambda\right)^{2}+\Delta^{2}}}=\frac{2}{G}, \\
\sum_{a}\left(j_{a}+\frac{1}{2}\right)\left[1-\frac{\varepsilon_{a}-\lambda}{\sqrt{\left(\varepsilon_{a}-\lambda\right)^{2}+\Delta^{2}}}\right]=N,
\end{gathered}
$$

where $\lambda, \Delta$, and $G$ represent the Fermi energy, pairing gap, and the pairing force constant, respectively. $N$ is the number of neutrons or protons involved in the pairing correlations. The solution of those two coupled equations, Eq.(20) and (21) allows one to find $\lambda$ and $\Delta$. The contribution of the pairing interaction to the total energy can be expressed as following:

$$
E_{\text {pair }}=-G\left[\sum_{a}\left(j_{a}+\frac{1}{2}\right) v_{a} u_{a}\right]^{2},
$$

where $v_{a}^{2}$ is the occupation probability of a state with quantum numbers $\alpha=\left(n, l, j, m, \tau_{3}\right) \equiv$ $\left(a, m, \tau_{3}\right)$ and $u_{a}^{2}=1-v_{a}^{2}$. For spherical nuclei, the eigenvalues of Dirac equations are of degeneracy for magnetic quantum numbers $m$ within the same $(n, l, j)$. One has:

$$
\left(\begin{array}{c}
v_{a}^{2} \\
u_{a}^{2}
\end{array}\right)=\frac{1}{2}\left(1 \mp \frac{\varepsilon_{a}-\lambda}{\sqrt{\left(\varepsilon_{a}-\lambda\right)^{2}+\Delta^{2}}}\right),
$$

In principle, the continuous spectrum is also the solution of Dirac Eq.(9), both continuous and bound states constitute a complete set of basis. In most previous theoretical nuclear structure calculations, single particle states in the continuum are usually treated in a discretization procedure by expanding wave functions in a set of harmonic oscillator basis or setting a box. This approximation, however, can be justified for very narrow resonances and gives a global description of the contributions from the continuum. In this work we introduce single particle resonant states into the pairing gap equations instead of the discretized continuous states in order to investigate the width effect of resonances on pairing correlations. The resonant state wave function are obtained by imposing a scattering boundary condition. At the distance $\mathrm{R}$ where the nuclear potentials vanish, the upper component of the neutron radial wave function has the following asymptotic behavior:

$$
G_{\nu}(k r)=A_{\nu}\left[j_{l_{\nu}}(k r)-\tan \delta_{\nu} n_{l_{\nu}}(k r)\right], \quad \text { for } r \geq R
$$


where $j_{l_{\nu}}$ and $n_{l_{\nu}}$ are spherical Bessel and Neumann functions, respectively, and $\delta_{\nu}$ is the phase shift corresponding to the angular momentum $\left(l_{\nu}, j_{\nu}\right), k^{2}=E^{2}-M^{2}$. For the case of proton, the asymptotic behavior can be obtained by replacing the spherical Bessel and Neumann functions in Eq.(24) with the relativistic regular and irregular Coulomb wave functions [25], respectively. The energy of a resonant state is determined when the phase shift of the scattering state reaches $\pi / 2$. The wave function of scattering state is normalized to a delta function of energy $\delta\left(E-E^{\prime}\right)$. This condition fixes the normalization constant $A_{\nu}$, it can be obtained as:

$$
A_{\nu}=\cos \delta_{\nu} \sqrt{\frac{1}{\pi} \frac{M+E}{2 k}},
$$

In order to take into account the width effect of resonant states in the continuum we introduce a level density of the continuum [26] into the gap equations. When we introduce single particle resonant states with widths into the pairing correlations, the gap equations can be expressed as:

$$
\begin{gathered}
\sum_{a}\left(j_{a}+\frac{1}{2}\right) \frac{1}{\sqrt{\left(\varepsilon_{a}-\lambda\right)^{2}+\Delta^{2}}}+\sum_{\nu}\left(j_{\nu}+\frac{1}{2}\right) \int_{I_{\nu}} \frac{g_{\nu}\left(\varepsilon_{\nu}\right)}{\sqrt{\left(\varepsilon_{\nu}-\lambda\right)^{2}+\Delta^{2}}} d \varepsilon_{\nu}=\frac{2}{G}, \\
\sum_{a}\left(j_{a}+\frac{1}{2}\right)\left[1-\frac{\varepsilon_{a}-\lambda}{\sqrt{\left(\varepsilon_{a}-\lambda\right)^{2}+\Delta^{2}}}\right]+\sum_{\nu}\left(j_{\nu}+\frac{1}{2}\right) \int_{I_{\nu}} g_{\nu}\left(\varepsilon_{\nu}\right)\left[1-\frac{\varepsilon_{\nu}-\lambda}{\sqrt{\left(\varepsilon_{\nu}-\lambda\right)^{2}+\Delta^{2}}}\right] d \varepsilon_{\nu}=N .
\end{gathered}
$$

The sums $a$ and $\nu$ run over the bound states and resonant states involved in the pairing calculation, respectively, and $I_{\nu}$ is an energy interval associated with each partial wave $\left(l_{\nu}, j_{\nu}\right)$. The factor $g_{\nu}$ is defined as:

$$
g_{\nu}\left(\varepsilon_{\nu}\right)=\frac{1}{\pi} \frac{d \delta_{\nu}}{d \varepsilon_{\nu}}
$$

which is the so-called continuum level density and $\delta_{\nu}$ is the phase shift of the scattering state with angular momentum $\nu=\left(l_{\nu}, j_{\nu}\right)$. The factor $g_{\nu}$ represents the variation of the localization of scattering states in the energy region of a resonance, in other words, it reflects the widths effect of the resonant states. For a very narrow resonant state, the factor $g_{\nu}$ becomes a delta function.

Taking account of those resonant continuum in the gap equations, the expressions of various densities in Eq.(16 - 19) have to be modified. They can be expressed as: 


$$
\begin{aligned}
\rho_{s}(r)= & \sum_{a} \frac{2 j_{a}+1}{4 \pi} v_{a}^{2}\left(\left|G_{a}(r)\right|^{2}-\left|F_{a}(r)\right|^{2}\right) \\
& +\sum_{\nu} \frac{2 j_{\nu}+1}{4 \pi} \int_{I_{\nu}}\left(\left|G_{\nu}(r)\right|^{2}-\left|F_{\nu}(r)\right|^{2}\right) g_{\nu}\left(\varepsilon_{\nu}\right) v_{\nu}^{2}\left(\varepsilon_{\nu}\right) d \varepsilon_{\nu} \\
\rho_{v}(r)= & \sum_{a} \frac{2 j_{a}+1}{4 \pi} v_{a}^{2}\left(\left|G_{a}(r)\right|^{2}+\left|F_{a}(r)\right|^{2}\right) \\
& +\sum_{\nu} \frac{2 j_{\nu}+1}{4 \pi} \int_{I_{\nu}}\left(\left|G_{\nu}(r)\right|^{2}+\left|F_{\nu}(r)\right|^{2}\right) g_{\nu}\left(\varepsilon_{\nu}\right) v_{\nu}^{2}\left(\varepsilon_{\nu}\right) d \varepsilon_{\nu} \\
\rho_{3}(r)= & \sum_{a} \frac{2 j_{a}+1}{4 \pi} \tau_{3} v_{a}^{2}\left(\left|G_{a}(r)\right|^{2}+\left|F_{a}(r)\right|^{2}\right) \\
& +\sum_{\nu} \frac{2 j_{\nu}+1}{4 \pi} \tau_{3} \int_{I_{\nu}}\left(\left|G_{\nu}(r)\right|^{2}+\left|F_{\nu}(r)\right|^{2}\right) g_{\nu}\left(\varepsilon_{\nu}\right) v_{\nu}^{2}\left(\varepsilon_{\nu}\right) d \varepsilon_{\nu} \\
\rho_{c}(r)=\sum_{a} & \frac{2 j_{a}+1}{4 \pi}\left(\frac{1-\tau_{3}}{2}\right) v_{a}^{2}\left(\left|G_{a}(r)\right|^{2}+\left|F_{a}(r)\right|^{2}\right) \\
+\sum_{\nu} & \frac{2 j_{\nu}+1}{4 \pi}\left(\frac{1-\tau_{3}}{2}\right) \int_{I_{\nu}}\left(\left|G_{\nu}(r)\right|^{2}+\left|F_{\nu}(r)\right|^{2}\right) g_{\nu}\left(\varepsilon_{\nu}\right) v_{\nu}^{2}\left(\varepsilon_{\nu}\right) d \varepsilon_{\nu} \\
& +
\end{aligned}
$$

The Dirac equation Eq.(9) and meson field functions (12 - 14) as well as the photon field function (15) with corresponding densities (29-32) are solved self-consistently in an iterative way. Therefore, the total binding energy can be written as:

$$
\begin{aligned}
E= & \sum_{a}\left(2 j_{a}+1\right) v_{a}^{2} E_{a}+\sum_{\nu}\left(2 j_{\nu}+1\right) \int_{I_{\nu}} g_{\nu}\left(\varepsilon_{\nu}\right) v_{\nu}^{2}\left(\varepsilon_{\nu}\right) \varepsilon_{\nu} d \varepsilon_{\nu} \\
& -\frac{1}{2} \int\left(g_{\sigma} \rho_{s} \sigma_{0}+g_{\omega} \rho_{\nu} \omega_{0}+g_{\rho} \rho_{3} \rho_{0}^{3}+e \rho_{c} A_{0}\right) d^{3} r \\
& -\int\left(\frac{1}{3} g_{2} \sigma_{0}^{3}+\frac{1}{4} g_{3} \sigma_{0}^{4}\right) d^{3} r \\
& -G\left[\sum_{a}\left(j_{a}+\frac{1}{2}\right) v_{a} u_{a}+\sum_{\nu}\left(j_{\nu}+\frac{1}{2}\right) \int_{I_{\nu}} g_{\nu}\left(\varepsilon_{\nu}\right) v_{\nu}\left(\varepsilon_{\nu}\right) u_{\nu}\left(\varepsilon_{\nu}\right) d \varepsilon_{\nu}\right]^{2} \\
& -\frac{3}{4} \cdot 41 \cdot A^{-1 / 3}
\end{aligned}
$$

Where the last two terms are the pairing energy and the correction for the spurious center of mass motion, respectively. 


\section{PROPERTIES OF NEUTRON RICH EVEN-EVEN NI ISOTOPES}

In this work we investigate the ground state properties of neutron rich even-even $\mathrm{Ni}$ isotopes in the RMF+BCS model and explicitly take account of the continuum. Particularly we shall focus our attention on the effect of single particle resonant states on the pairing correlation in those neutron rich nuclei. Calculations are carried out in the RMF with the parameter set NL3. Three types of calculations: RMF+BCS, RMF+BCSR and RMF + BCSRW are performed. The effect of single particle states in the continuum on the ground state properties is investigated.

In our investigation, the proton number of $\mathrm{Ni}$ isotopes is $Z=28$, which is a closed shell. Therefore, the proton pairing gap is taken to be zero. For the neutron case, we use a stateindependent pairing strength $G=C / A$, where the constant $C=20.5 \mathrm{MeV}$ is adopted from Ref.[14]. It could also provide a best fit to reproduce experimental binding energies of known $\mathrm{Ni}$-isotopes. In practical calculations, we restrict the pairing space to about one harmonic oscillator shell above and below the Fermi surface in the RMF+BCS model. The levels include $1 f_{5 / 2}, 2 p_{3 / 2}, 2 p_{1 / 2}, 1 g_{9 / 2}, 2 d_{5 / 2}, 3 s_{1 / 2}, 2 d_{3 / 2}, 1 g_{7 / 2}, 1 h_{11 / 2}$ and $2 f_{7 / 2}$, some of them, such as $2 d_{5 / 2}, 2 d_{3 / 2}, 1 g_{7 / 2}, 2 f_{7 / 2}$, and $1 h_{11 / 2}$ are single particle resonant states, depending on the isotopes. Highly excited resonant states with large widths, such as $1 h_{9 / 2}$ and $1 i_{13 / 2}$, are ignored in our calculations. Obviously, an enlarged pairing space in the normal BCS calculation would change the pairing contribution due to a constant pairing interaction. Therefore the results in cases of RMF+BCS and RMF+BCSR in Fig.1 and Fig.2 would be different if one includes more states in the pairing active space. It is found that the pairing properties are not affected too much if one includes the resonant states $1 h_{9 / 2}$ and $1 i_{13 / 2}$ with wide widths in the RMF+BCSRW calculation.

The single particle energies and wave functions are first carried out by solving the Dirac equation Eq.(9) self-consistently. Using those single particle states we can solve the BCS gap equations in the three approaches. The Fermi energy and gap as well as the occupation probabilities of quasi-particle states are obtained simultaneously. The nuclear densities composed of quasi-particle states and potentials are recalculated. Then we solve Dirac equation again by an iterative way until the convergence is reached. The pairing correlation energies can be obtained in the Eq.(22) in the three approaches, which are shown in Fig.1 for neutron rich even-even $\mathrm{Ni}$ isotopes of $N=40$ to 70 . Pronounced differences of the 
pairing energies performed in various $\mathrm{RMF}+\mathrm{BCS}$ approaches are observed for open shell nuclei, where the behavior of pairing energies is similar to those obtained by the RHB in Refs. [27]. The usual RMF+BCS approach produces the largest pairing energies, due to some non-resonant scattering states in the continuum are included. It can be seen in Fig.1 that the pairing energies are reduced largely when the widths of single particle resonant states are taken into account in the RMF+BCSRW calculation.

In order to understand the difference presented by different treatments on pairing correlations, we plot the results of pairing gaps and Fermi energies for neutron rich even-even $\mathrm{Ni}$ isotopes with masses from 68 to 98 in Fig.2(a) and Fig.2(b). The curve in Fig.2 (a) is the empirical pairing gap given by $11.2 / \sqrt{A}$. It is observed that pairing gaps are largely decreased when the width effect of single particle resonant states is taken in account in pairing correlation calculations, while the Fermi energies remain unchanged for three approaches. This feature agrees with the case in the non-relativistic HF+BCS calculations[10]. It is found that the pairing gaps in the RMF+BCSR calculation are close to those obtained in the box RMF+BCS approximation. As an example, for the neutron rich nucleus ${ }^{90} \mathrm{Ni}$, the pairing gap and Fermi energy are $\Delta_{n}=1.558 \mathrm{MeV}$ and $\lambda_{n}=-1.278 \mathrm{MeV}$ in the RMF+BCSR approach, whereas, they are reduced to $1.223 \mathrm{MeV}$ and $-1.256 \mathrm{MeV}$, respectively when we perform the pairing correlation calculations by considering the width effect of resonances explicitly. The reduced pairing gap and Fermi energy could change the occupation probability of neutrons at each single particle orbit near the Fermi surface. In Fig.3 we show the occupation probabilities of neutron single particle levels in ${ }^{90} \mathrm{Ni}$ as a function of corresponding energies. The arrow is the position of the neutron Fermi surface. In ${ }^{90} \mathrm{Ni}$ the Fermi energy is very close to the continuum, therefore the continuum becomes important in the pairing correlations. Some scattering states in the continuum, such as states $3 p_{3 / 2}$ and $3 p_{1 / 2}$, which are not resonance states due to the low centrifugal barrier, are included in the RMF+BCS calculation. Therefore the RMF+BCS approach overestimates the pairing correlation and produces large pairing energies and pairing gaps. It is found that the width effect on the pairing is to reduce the pairing correlations, therefore occupation probabilities of low-lying states below the Fermi surface in the case of RMF+BCSRW are slightly larger than those without the width effect, and vice versa for states above Fermi surface. 
In Fig. 4 we display the two-neutron separation energy $S_{2 n}$ :

$$
S_{2 n}=B(Z, N)-B(Z, N-2) .
$$

We calculate two-neutron separation energies for $\mathrm{Ni}$ isotopes till the neutron drip line in three approaches, RMF+BCS, RMF+BCSR and RMF+BCSRW. The two-neutron separation energies calculated in the RMF without pairings are also plotted in Fig.4, which are denoted by crosses. The empirical data of $S_{2 n}$ are obtained by Eq.(34), where the experimental binding energies of the Ni isotopes for $N \leq 50$ are taken from the Ref.[28]. The theoretical binding energies for $\mathrm{Ni}$ isotopes are listed in Table I. The position of the neutron drip line may be determined by the signature where the two-neutron separation energy changes its sign or the Fermi energy becomes positive. It is found that the position of the neutron drip line predicted by various RMF+BCS models is located between ${ }^{98} \mathrm{Ni}$ and ${ }^{100} \mathrm{Ni}$, the same result is also obtained in RHB calculations [27]. It is found that the two-neutron separation energies calculated in various RMF+BCS approaches are very close to each other, which can well reproduce the experimental data in the region of $N=42$ to $N=50$. In contrast, the results without pairing considerably deviate from the others. It indicates that the pairing is responsible to a reasonable description of the two-neutron separation energy. Some disagreements with experimental data are found for $N=38$ and $N=40$ isotopes, which were also observed in the RHB calculation with the parameter set NL3 29, 30]. Although pairing energies obtained in these three approaches with different treatment of pairings differ significantly, two-neutron separation energies are consistent with each other. This is because that differences, which appear in the pairing correlation energy are largely cancelled in the $S_{2 n}$.

In table $\mathrm{I}$, it is shown that the binding energies produced in the three RMF+BCS approaches scatter when nuclei are far away from the $\beta$ stable line. To clearly illustrate the width effect of the resonant states on the pairings we define a new quantity, which characterizes the pairing correlations energy:

$$
E_{B C S}=E(R M F)-E(R M F+B C S)
$$

The $E_{B C S}$ of $\mathrm{Ni}$ isotopes calculated in various approaches are plotted in Fig.5. It is shown that the results produced in the three approaches are very close to each other for those isotopes not far away from the $\beta$ stable-line. It implies that the width of the resonance in 
the pairing correlation could be neglected at $N \leq 34$. The RMF $+\mathrm{BCS}$ and RMF $+\mathrm{BCSR}$ give more or less similar values of $E_{B C S}$, even near the drip line. The width effect gets more and more pronounced as the neutron number increases, especially near the drip line. Therefore a proper treatment of the resonant continuum including its width might be necessary for the nuclei near the drip line.

Neutron rms radii of neutron rich even-even $\mathrm{Ni}$ isotopes are further calculated in the three approaches, which are plotted in Fig.6. The rms radii calculated in the RMF are also shown in Fig.6 and displayed by cross. It is seen that neutron rms radii of $\mathrm{Ni}$ isotopes at $A<80$ calculated in all approaches are very close to each other, which is similar to what was observed in the binding energy. The neutron rms radii calculated in the RMF+BCS with a box become larger at $A>80$ in comparison with those produced in other approaches. This is due to the fact that the contribution of the pairing correlation from the scattering states $3 p_{3 / 2}$ and $3 p_{1 / 2}$ is included in the RMF+BCS calculations. They are not resonant states and their wave functions are not well localized inside the nucleus. The neutron rms radius for ${ }^{84} \mathrm{Ni}$ given in the RMF is slightly smaller than that obtained in the other approaches. Actually this is a natural result when the pairing correlation is switched on. The neutron rms radius in the $\mathrm{RMF}+\mathrm{BCS}$ is increased because the pairing interaction scatters some neutrons from $2 d_{5 / 2}$ to the loosely bound state $3 s_{1 / 2}$ which is a state less localized. In contrast, in the case of ${ }^{90} \mathrm{Ni}$ the states $3 s_{1 / 2}$ and $2 d_{3 / 2}$, which are completely occupied in the RMF approximation are scattered to states $1 g_{7 / 2}$ and $1 h_{11 / 2}$ due to the pairing interaction. Although the states $1 g_{7 / 2}$ and $1 h_{11 / 2}$ are close to or buried in the continuum, their wave functions are more localized inside the nucleus than those for the $3 s_{1 / 2}$ and $2 d_{3 / 2}$ states due to a high centrifugal barrier. Therefore the rms radius of ${ }^{90} \mathrm{Ni}$ calculated in the RMF is larger than that produced in calculations including the pairing correlation.

We plot the neutron density of ${ }^{84} \mathrm{Ni}$ in Fig.7, where the dash-dotted curve is obtained in the RMF, the short-dotted, dashed and solid curves are produced in the RMF+BCS, RMF +BCSR and RMF +BCSRW approaches, respectively. The tail of the density distribution gets larger when the pairing correlation is considered. The width effect on the density distribution is very small, which is consistent to the calculated neutron rms radii. In order to show the effect of the box size on the density in the box RMF+BCS approximation we also plot the neutron density with various values of the box size $R_{\text {box }}=15,20,25 \mathrm{fm}$ in Fig.7. It is observed that the tail of the density strongly depends on the box size, where 
unphysical particle gas may appear in the exotic nucleus. In the RMF+BCS calculation non-resonant discretized states in the continuum are included in pairing correlation, such as $3 p_{3 / 2}$ and $3 p_{1 / 2}$ states. Their wave functions have a long tail strongly depending on the box size. It is found that this problem is well overcome when one performs the pairing correlation calculation with only a few narrow resonant states in stead of those discretized states in the continuum.

\section{SUMMARY}

In this paper, we have investigated the pairing correlation for neutron-rich $\mathrm{Ni}$ isotopes in the relativistic mean field theory and Bardeen-Cooper-Schrieffer approximation with a constant pairing strength. A proper treatment of the single particle resonant state in the continuum on pairing correlations has to include not only its energy, but also its width. The inclusion of the width of the resonant state in the pairings can be performed by introducing a level density in the continuum into the pairing gap equation. The resonant continuum is solved by imposing a proper scattering boundary condition. The investigation is performed in three approaches: $\mathrm{RMF}+\mathrm{BCS}, \mathrm{RMF}+\mathrm{BCSR}$ and $\mathrm{RMF}+\mathrm{BCSRW}$ with the effective Lagrangian parameter set NL3. A special attention is paid on the width effect of resonant states in the continuum on the pairing correlation for nuclei close to the drip line. We have studied the width effect of the resonant continuum on pairings: pairing gap, Fermi energy and occupation probability, as well as nuclear ground state properties, such as binding energy, two-neutron separation energy, neutron rms radius and neutron density. The results show that a proper treatment of the resonant continuum in pairing correlations, which explicitly includes the width of the single particle resonant state is important for nuclei close to neutron drip line. They could affect the pairing gaps, Fermi energies, pairing energies, and binding energies considerably. Various RMF+BCS approaches could give a similar description on the ground state properties for nuclei not far away from the $\beta$ stable line. It is observed that unphysical particle gas, which may appear in the traditional mean field plus BCS calculation for nuclei in the vicinity of drip line can be well overcome when one performs pairing correlation calculations with only resonant states instead of discretized states in the continuum. It might be concluded that the simple BCS method works well in describing the pairing correlations for neutron rich nuclei provided the continuum is properly 
treated.

\section{Acknowledgments}

One of the authors (L.G. Cao) wishes to thank Prof. Zhang Zong-ye and Prof. Yu You-wen for many stimulating discussions. This work is supported by the National Natural Science Foundation of China under Grant Nos 10305014, 90103020, 10075080 and 10275094, and Major State Basic Research Development Programme in China under Contract No G2000077400.

[1] P. Ring, Prog. Part. Nucl. Phys. 37, 197 (1996).

[2] J. Dobaczewski, W. Nazarewicz, Phil. Trans. R. Soc. Lod. A356, 2007 (1998).

[3] T. T. S. Kuo, F. Krmpotic, and Y. Tzeng, Phys. Rev. Lett. 78, 2708 (1997).

[4] J. Dobaczewski, W. Nazarewicz, T. R. Werner, J. F. Berger, C. R. Chinn and J. Dechargé, Phys. Rev. C53, 2809 (1996).

[5] P. Ring and P. Schuck, "The Nuclear Many-Body Problem" (Springer-Verlag, Berlin, 1980).

[6] Jun-Qing Li ,Zhong-Yu Ma, Bao-Qiu Chen, Yong Zhou, Phys. Rev. C65, 064305 (2002).

[7] J. Dobaczewski, H. Flocard, and J. Treiner, Nucl. Phys. A422, 103 (1984).

[8] H. Kucharek and P.Ring, Z. Phys. A339, 23 (1991).

[9] N. Sandulescu, R. J. Liotta, and R. Wyss, Phys. Lett. B394, 6 (1997).

[10] N. Sandulescu, Nguyen Van Giai, and R. J. Liotta, Phys. Rev. C61, 061301(R) (2000).

[11] A. T. Kruppa, P. H. Heenen and R. J. Liotta, Phys. Rev. C63 044324 (2001).

[12] N. Sandulescu, L.S. Geng, H. Toki, and G. Hillhouse, Phys. Rev. C68 054323 (2003).

[13] Li-Gang Cao and Zhong-Yu Ma, Phys. Rev. C66, 024311 (2002).

[14] M. Del Estal, M. Centelles, X. Viñas, and S. K. Patra, Phys. Rev. C63, 044321 (2001).

[15] G. A. Lalazissis, J.König and P.Ring, Phys. Rev. C55, 540 (1997).

[16] G. A. Lalazissis, S. Raman and P. Ring, At. Data Nucl. Data Tables 71, 1 (1999).

[17] Zhong-yu Ma, A. Wandelt, N. V. Giai, D. Vretenar, P. Ring, Nucl. Phys. A686, 173 (2001).

[18] Zhong-yu Ma, A. Wandelt, N. V. Giai, D. Vretenar, P. Ring, and Li-gang Cao, Nucl. Phys. A703, $222(2002)$. 
[19] P. Ring, Zhong-yu Ma, N. V. Giai, A. Wandelt, D. Vretenar, and Li-gang Cao, Nucl. Phys. A694, 249 (2001).

[20] Li-Gang Cao and Zhong-Yu Ma, Chin. Phys. Lett. 20, 1459 (2003).

[21] C. J. Horowitz and B. D. Serot, Nucl. phys. A 368, 503 (1981).

[22] B. D. Serot and J. D. Walecka, Adv. Nucl. Phys. 16, 1 (1986).

[23] J. Boguta, and A. R. Bodmer, Nucl. Phys. A292, 413 (1977).

[24] M. Grasso, N. Sandulescu, Nguyen Van Giai, and R. J. Liotta, Phys. Rev. C64, 064321 (2001).

[25] W. Greiner, "Relativistic Quantum Mechanics - Wave Equation" (Springer-Verlag 1997).

[26] P. Bonche, S. Levit, and D. Vautherin, Nucl. Phys. A427, 278 (1984).

[27] J. Meng, Phys. Rev. C57, 1229 (1998).

[28] G. Audi and A.H. Wapstra, Nucl.Phys. A595, 409 (1995).

[29] G. A. Lalazissis, D. Vretenar, and P. Ring,Phys. Rev. C57, 2294 (1998).

[30] M. M. Sharma, A. R. Farhan, and S. Mythili, Phys. Rev. C61, 054306 (2000). 
TABLE I: The binding energies of $\mathrm{Ni}$ isotopes near the neutron drip line calculated in the RMF + BCS, RMF+BCSR, and RMF+BCSRW approaches with parameter set NL3. The available experimental binding energies of the $\mathrm{Ni}$ isotopes for $N \leq 50$ from Ref. 28] are also shown. All energy values in the table are in unit of $\mathrm{MeV}$.

\begin{tabular}{|c|c|c|c|c|}
\hline & $\mathrm{RMF}+\mathrm{BCS}$ & $\mathrm{RMF}+\mathrm{BCSR}$ & RMF+BCSRW & Exp. \\
\hline${ }^{58} \mathrm{Ni}$ & 502.347 & 502.275 & 502.104 & 506.453 \\
\hline${ }^{60} \mathrm{Ni}$ & 521.655 & 521.448 & 521.363 & 526.841 \\
\hline${ }^{62} \mathrm{Ni}$ & 540.458 & 540.374 & 540.251 & 545.258 \\
\hline${ }^{64} \mathrm{Ni}$ & 558.361 & 558.273 & 558.055 & 561.754 \\
\hline${ }^{66} \mathrm{Ni}$ & 575.642 & 575.579 & 575.009 & 576.830 \\
\hline${ }^{68} \mathrm{Ni}$ & 590.892 & 590.960 & 590.930 & 590.430 \\
\hline${ }^{70} \mathrm{Ni}$ & 603.233 & 603.195 & 602.775 & 602.600 \\
\hline${ }^{72} \mathrm{Ni}$ & 614.246 & 614.116 & 613.567 & 613.900 \\
\hline${ }^{74} \mathrm{Ni}$ & 624.428 & 624.307 & 623.752 & 623.900 \\
\hline${ }^{76} \mathrm{Ni}$ & 633.840 & 633.816 & 633.414 & 633.100 \\
\hline${ }^{78} \mathrm{Ni}$ & 642.352 & 642.555 & 642.555 & 641.400 \\
\hline${ }^{80} \mathrm{Ni}$ & 647.837 & 647.659 & 646.964 & \\
\hline${ }^{82} \mathrm{Ni}$ & 652.279 & 651.943 & 650.888 & \\
\hline${ }^{84} \mathrm{Ni}$ & 656.092 & 655.678 & 654.472 & \\
\hline${ }^{86} \mathrm{Ni}$ & 659.422 & 658.975 & 657.682 & \\
\hline${ }^{88} \mathrm{Ni}$ & 662.284 & 661.863 & 660.582 & \\
\hline${ }^{90} \mathrm{Ni}$ & 664.756 & 664.319 & 663.047 & \\
\hline${ }^{92} \mathrm{Ni}$ & 667.756 & 666.456 & 665.254 & \\
\hline${ }^{94} \mathrm{Ni}$ & 668.562 & 668.319 & 667.144 & \\
\hline${ }^{96} \mathrm{Ni}$ & 670.133 & 670.183 & 669.332 & \\
\hline${ }^{98} \mathrm{Ni}$ & 671.382 & 671.605 & 671.605 & \\
\hline
\end{tabular}




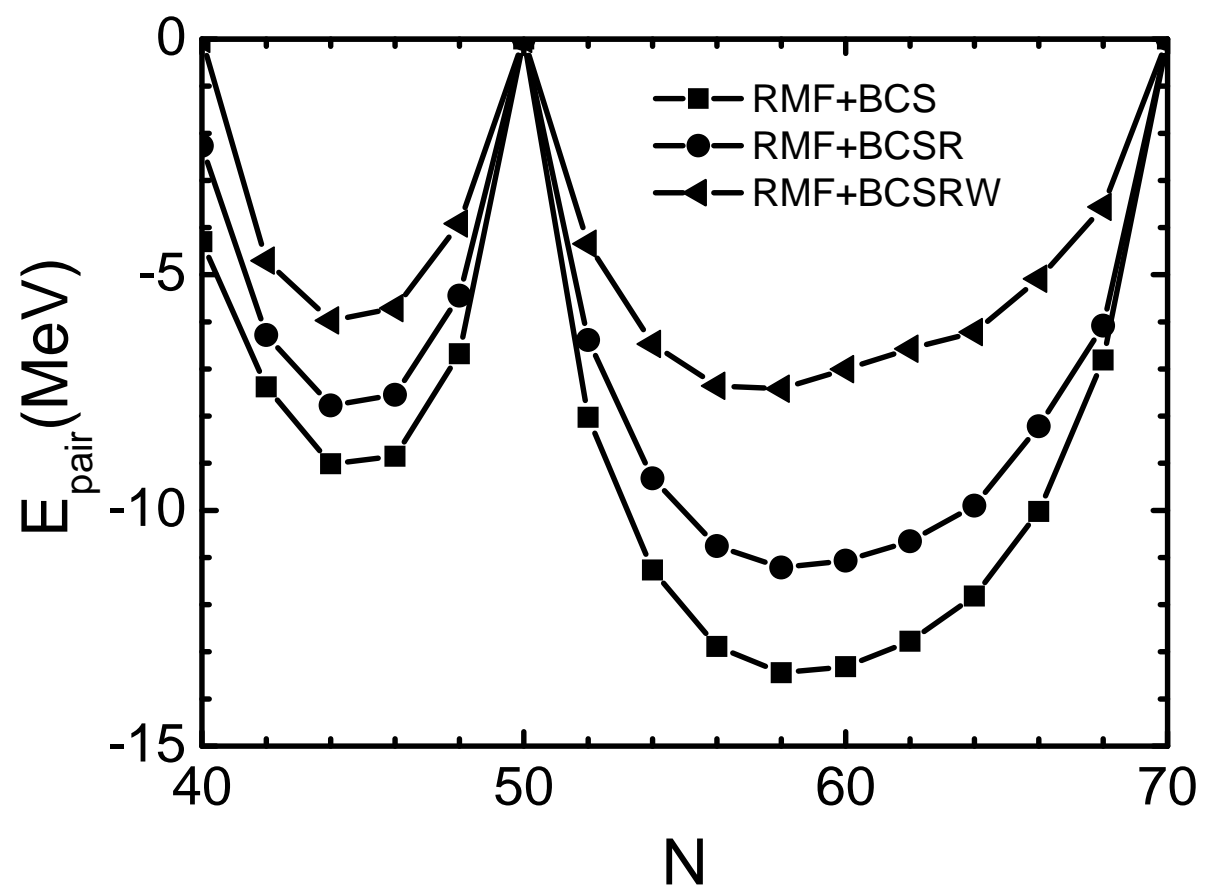

FIG. 1: The pairing energies for neutron rich even-even Ni isotopes with $N=40-70$. The solid squares, circles and left-triangles denote the results calculated in the RMF+BCS, RMF+BCSR and RMF+BCSRW approaches, respectively. All calculations are performed with the parameter set NL3. 

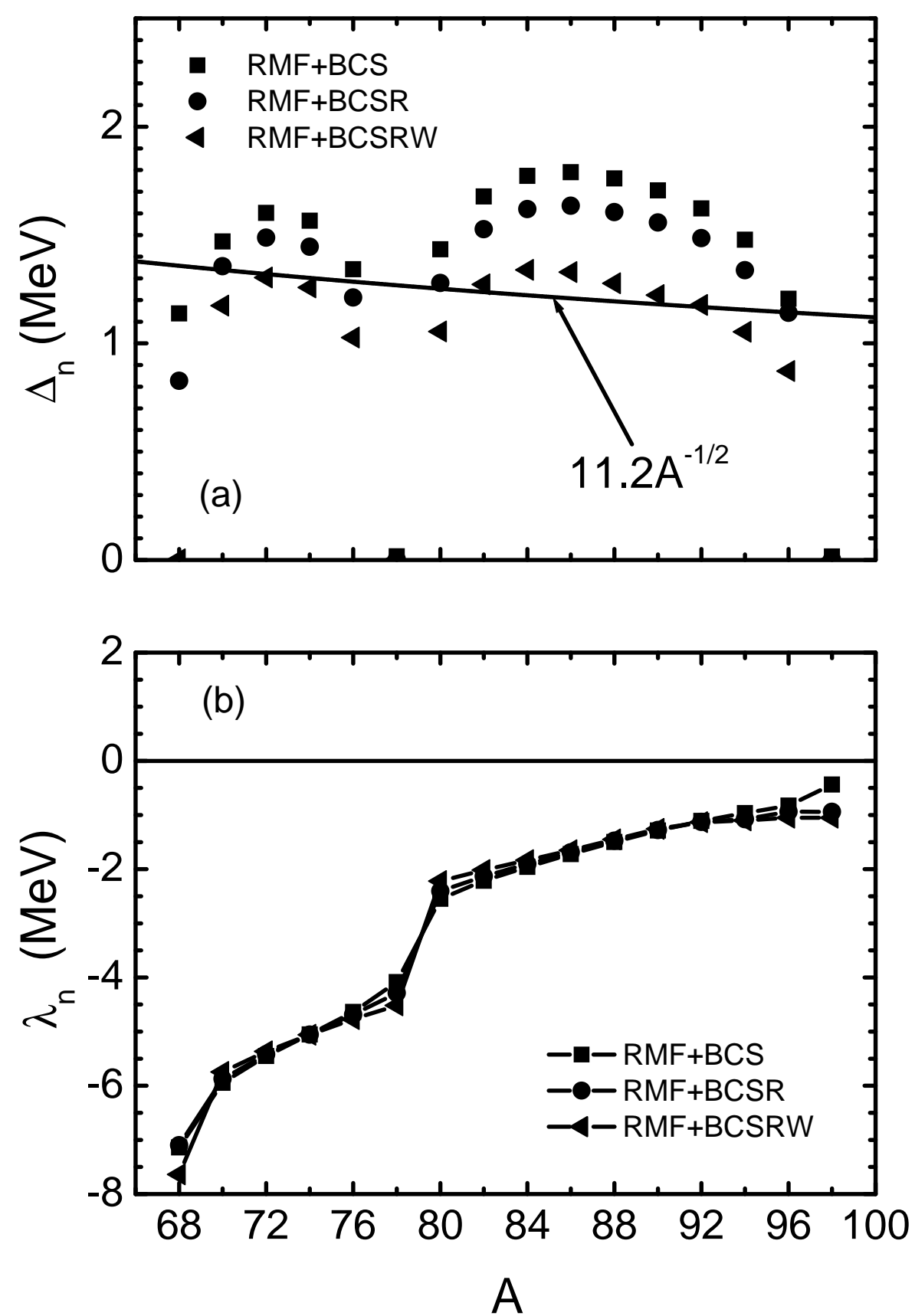

FIG. 2: The neutron pairing gap (a) and Fermi energy (b) as a function of the atomic number $A$. The notations are same as in Fig.1. 


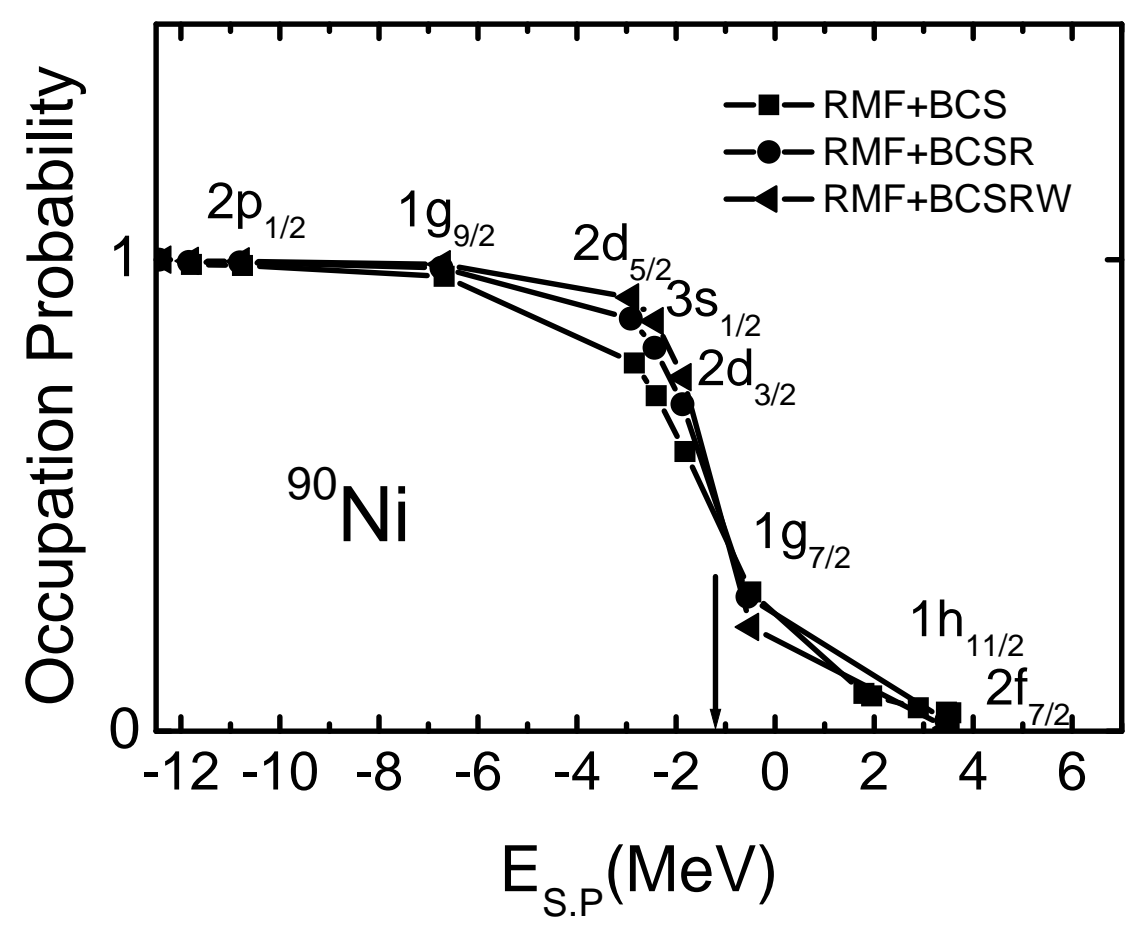

FIG. 3: The occupation probabilities for ${ }^{90} \mathrm{Ni}$ as a function of the single particle energy around the threshold. The results are performed in RMF+BCS, RMF+BCSR and RMF+BCSRW approaches with the effective nonlinear interaction NL3. The notations are same as in Fig.1. 


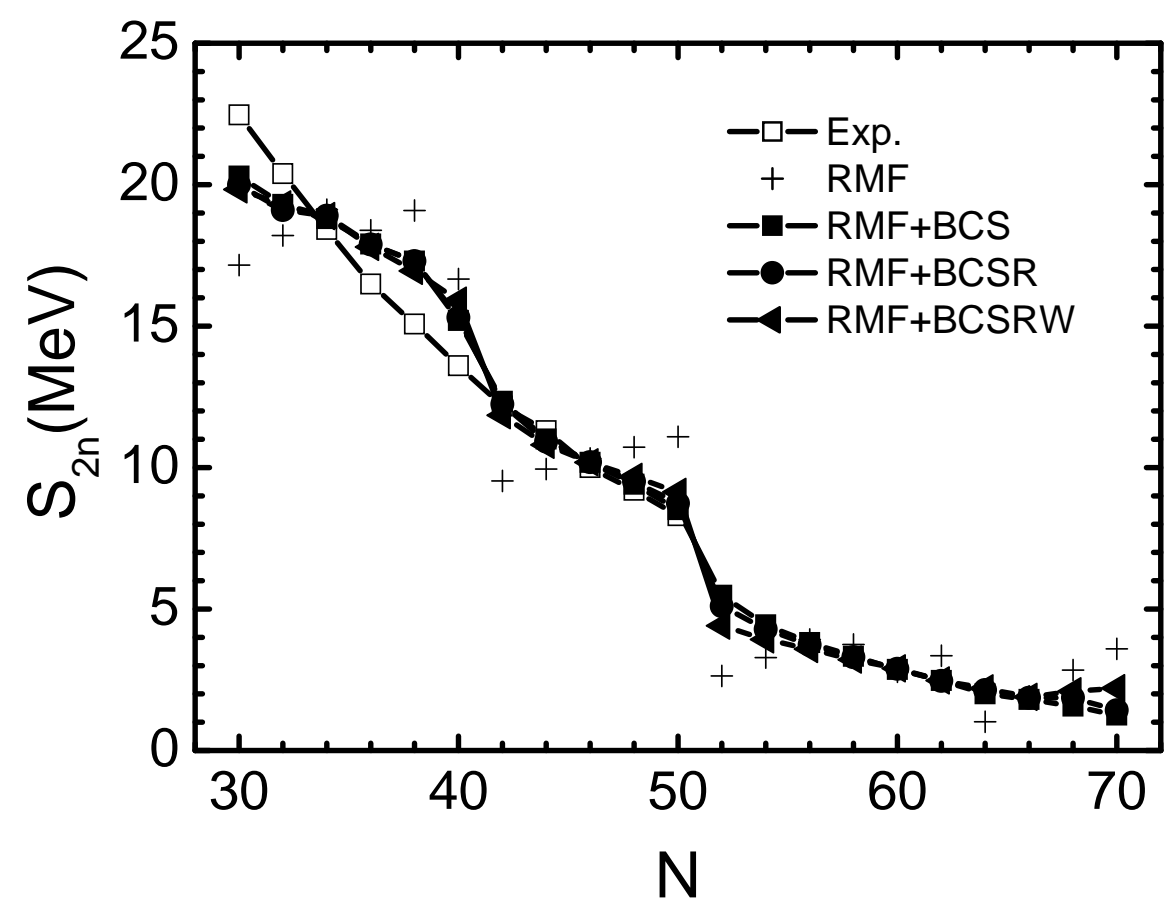

FIG. 4: Two-neutron separation energies for neutron rich even-even Ni isotopes calculated in the RMF, RMF+BCS, RMF+BCSR, and RMF+BCSRW approaches with parameter set NL3. The cross signs are the results calculated in the RMF. The experimental data denoted by open squares are taken from Ref.[28]. Other notations are same as in Fig.1. 


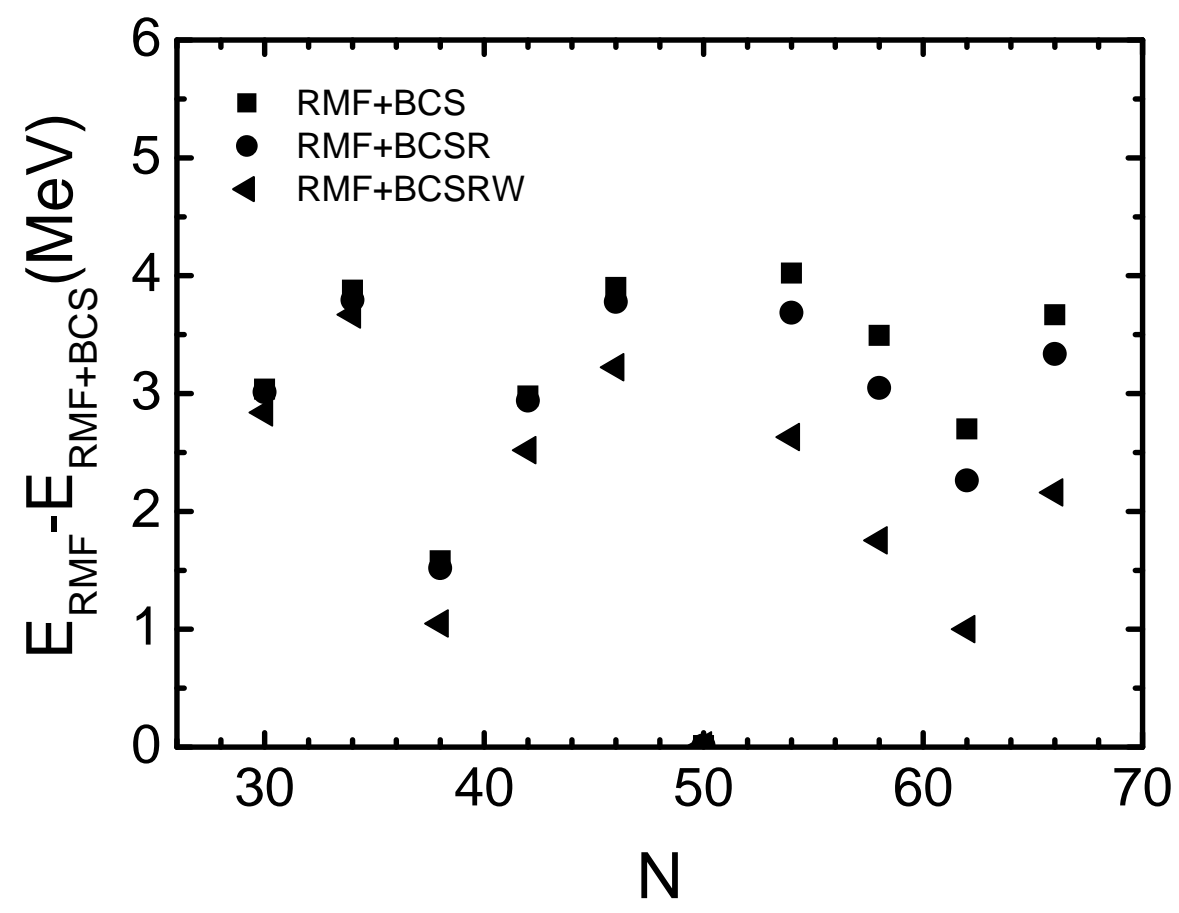

FIG. 5: The pairing correlation energies $E_{B C S}$ calculated in Eq.(35) in the RMF+BCS, RMF+BCSR and RMF+BCSRW approaches. The notations are same as in Fig.1. 


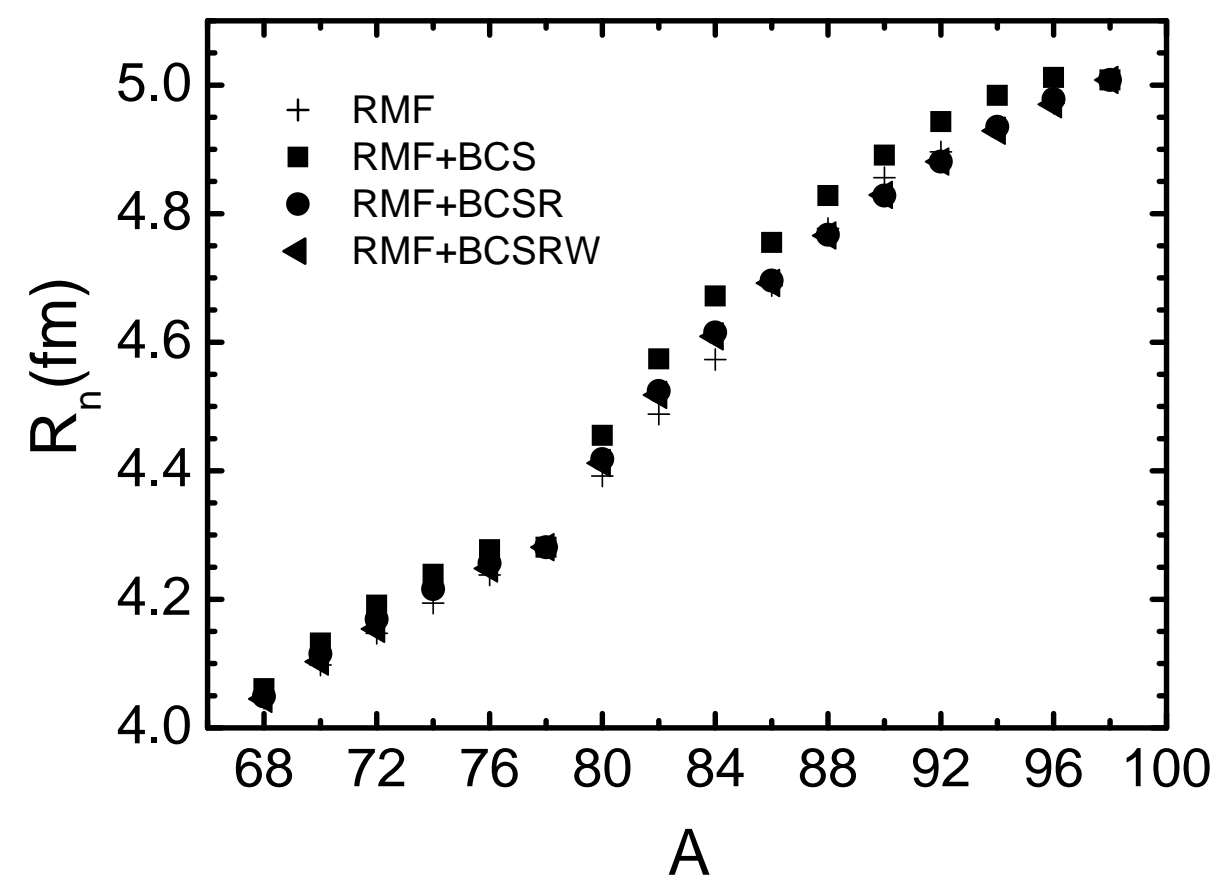

FIG. 6: Neutron rms radii for neutron rich even-even $\mathrm{Ni}$ isotopes calculated in the RMF, RMF+BCS, RMF+BCSR, and RMF+BCSRW approaches with parameter set NL3. The notations are same as in Fig.4 


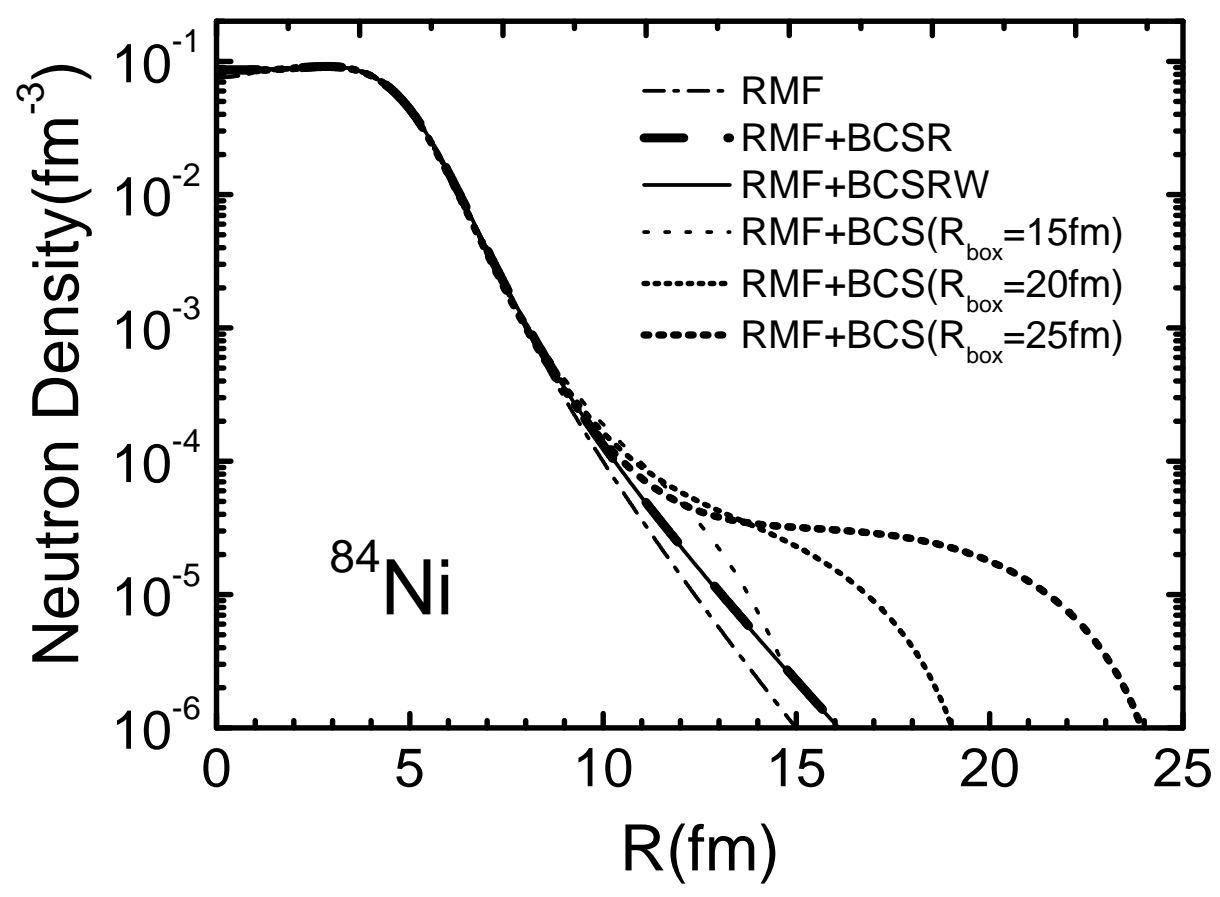

FIG. 7: Neutron density distribution for ${ }^{84} \mathrm{Ni}$ calculated in the RMF, RMF+BCS, RMF+BCSR, and RMF+BCSRW approaches, where the RMF+BCS results calculated with various values of the box size $\mathrm{R}_{\mathrm{box}}=15,20,25 \mathrm{fm}$ are also plotted. 